\title{
Neural Substrates of Abstinence-Induced Cigarette Cravings in Chronic Smokers
}

\author{
Ze Wang, ${ }^{1}$ Myles Faith, ${ }^{2}$ Freda Patterson, ${ }^{3}$ Kathy Tang, ${ }^{3}$ Kia Kerrin, ${ }^{3}$ E. Paul Wileyto, ${ }^{3}$ John A. Detre, ${ }^{1}$ and \\ Caryn Lerman ${ }^{3}$ \\ ${ }^{1}$ Department of Neurology, Center for Functional Neuroimaging, ${ }^{2}$ Weight and Eating Disorders Program, and ${ }^{3}$ Department of Psychiatry, Transdisciplinary \\ Tobacco Use Research Center, Abramson Cancer Center, University of Pennsylvania, Philadelphia, Pennsylvania 19104
}

Craving is a hallmark of drug dependence, including dependence on nicotine. Many studies have examined the neural substrates of cravings elicited by smoking-related cues. Less is known about the neural basis of unprovoked, abstinence-induced cravings, despite the contributions of such cravings to smoking relapse. To fill this gap, we used arterial spin labeled (ASL) perfusion magnetic resonance imaging to characterize the neural substrates of abstinence-induced cravings to smoke. Fifteen chronic smokers were scanned during a resting state on two separate occasions: (1) smoking satiety and (2) abstinence (after $\geq 12 \mathrm{~h}$ of smoking deprivation), in counterbalanced order. Smoking abstinence state (vs satiety) was associated with increased cerebral blood flow (CBF) in anterior cingulate cortex (ACC)/ medial orbitofrontal cortex (OFC) and left OFC. Abstinence-induced cravings to smoke were predicted by CBF increases (abstinence minus satiety) in the right OFC, right dorsolateral prefrontal cortex, occipital cortex, ACC, ventral striatum/nucleus accumbens, thalamus, amygdala, bilateral hippocampus, left caudate, and right insula. These data suggest that increased activation in the brain's visuospatial and reward circuitry underlies abstinence-induced cravings to smoke, and thereby, may be important in relapse.

Key words: addiction; cerebral blood flow; cortex; mesolimbic; nicotine; neuroimaging

\section{Introduction}

Cravings are a prominent feature of models of drug dependence, including nicotine, and a target of medication development efforts (Nestler, 2002; Lerman et al., 2007). However, not all forms of craving are equivalent with respect to the proximal causes or consequences. "Cue-elicited cravings" are thought to arise from a behavioral conditioning process in which stimuli associated with smoking trigger drug-seeking behavior (Caggiula et al., 2001; Conklin, 2006). A second form of craving develops rapidly after smoking cessation, in the absence of smoking-related cues (Jarvik et al., 2000). These "abstinence-induced cravings" appear to be more sensitive to effects of nicotine delivery than cue-induced cravings (Tiffany et al., 2000; Morissette et al., 2005). Furthermore, severity of abstinence-induced craving predicts lapses and relapse after a cessation attempt (Killen and Fortmann, 1997; Shiffman et al., 1997), whereas the relationship

Received June 29, 2007; revised 0ct. 12, 2007; accepted Nov. 3, 2007.

This work was supported by National Institutes of Health Grants R01 DA017555 (C.L.), R03DA023496 (Z.W.), P30 NS045839 and P41 RR02305 (J.A.D., Z.W.), and P50 CA/DA84718 (C.L.). This work was also supported in part by a grant from the Commonwealth of Pennsylvania Department of Health. The Department specifically disclaims responsibility for any analysis, interpretations, or conclusions. C.L., J.A.D., and M.F. were responsible for conceptualization and design of the study and manuscript preparation; Z.W., J.A.D., E.P.W., and K.T. for data analysis and manuscript preparation; and K.K. and F.P. for study implementation and data acquisition.

John A. Detre is an inventor of ASL and has received royalties from the University of Pennsylvania for its licensure. Correspondence should be addressed to either of the following: Dr. Ze Wang, Department of Neurology, Center for Functional Neuroimaging, University of Pennsylvania, 3400 Spruce Street, 3W Gates Building, Philadelphia, PA 19104, E-mail: zewang@mail.med.upenn.edu; or Dr. Caryn Lerman, Department of Psychiatry, Transdisciplinary Tobacco Use Research Center, University of Pennsylvania, 3535 Market Street, Suite 4100, Philadelphia, PA 19104, E-mail: clerman@mail.med.upenn.edu.

DOI:10.1523/JNEUROSCI.2966-07.2007

Copyright $\odot 2007$ Society for Neuroscience 0270-6474/07/2714035-06\$15.00/0 between cue-elicited craving and relapse is more equivocal (Niaura et al., 1989; Shadel et al., 1998).

The brain circuitry that underlies cue-elicited cravings to smoke has been explored extensively using positron emission tomography (PET) and functional magnetic resonance imaging (fMRI) (Brody, 2006). Compared with neutral cues, smokingrelated cues produce increased activation in the brain's visual attention and reward circuitry (Brody et al., 2002, 2004; David et al., 2005; McClernon et al., 2005). Some of these regions (e.g., dorsolateral prefrontal cortex, hippocampus, insula) are also implicated in subjective cravings in cue-induction studies (Brody et al., 2002; McClernon et al., 2005; Franklin et al., 2007). However, these studies varied with respect to the abstinence status of smokers, and only one (McClernon et al., 2005) compared cueinduced craving between abstinent and nonabstinent states. Thus, much less is known about how nicotine abstinence affects regional brain activation or about the neural circuitry underlying abstinence-induced cravings to smoke.

Predictions about the neural basis of abstinence-induced cravings can be generated based on previous neurobiological evidence. Nicotine binds to $\alpha 4 \beta 2$ nicotinic acetylcholine receptors (nAChRs) on dopaminergic neurons in the ventral tegmental area (Brody et al., 2006), which project to the nucleus accumbens, amygdala, and the prefrontal cortex (Kalivas and Volkow, 2005). In chronic smokers, a single cigarette produces nearly complete saturation and desensitization of $\alpha 4 \beta 2 \mathrm{nAChRs}$ (Brody et al., 2006). In contrast to nicotine delivery, abstinence from nicotine increases the availability of unbound $\alpha 4 \beta 2 \mathrm{nAChRs}$, which in turn, contributes to smoking urges (Staley et al., 2006). Thus, brain regions rich in $\alpha 4 \beta 2 \mathrm{nAChRs} \mathrm{(e.g.,} \mathrm{the} \mathrm{ventral} \mathrm{striatum,}$ 
cerebral cortex, thalamus) would be expected to exhibit changes in activation during nicotine abstinence versus satiety (Staley et al., 2006). Other regions of interest suggested by previous research include those important in neural plasticity, learning, and memory (e.g., the hippocampus, amygdala) (Ji et al., 2001; Tronson and Taylor, 2007), as well as attention and behavioral control [e.g., anterior cingulate cortex (ACC), medial orbitofrontal cortex (OFC)] (Rushworth et al., 2007).

To explore the neural basis of abstinence-induced cravings, smokers in the present study were scanned, using arterial spin labeled (ASL) perfusion magnetic resonance imaging (MRI), on two separate occasions: during a resting state of smoking satiety ( $1 \mathrm{~h}$ after cigarette consumption) versus a resting abstinent state (at least $12 \mathrm{~h}$ after cigarette consumption). The cerebral blood flow (CBF) changes associated with smoking abstinence (compared with satiety) were further examined for correlations with subjective abstinence-induced craving.

\section{Materials and Methods}

Subjects. All procedures were approved by the University of Pennsylvania Institutional Review Board. Fifteen smokers, 18-55 years old, who smoked at least 10 cigarettes per day for the last 6 months were recruited through newspaper advertisements. Persons with chronic medical, neurological, or Diagnostic and Statistical Manual of Mental Disorders, Ed IV, Axis I psychiatric disorder were excluded. Eligible participants were required to abstain from psychotropic medications for $14 \mathrm{~d}$ before the session and throughout the study (i.e., monoamine oxidase inhibitors, benzodiazepines, antidepressants, antipsychotics).

Design and procedures. The experiment used a within-subject design with two imaging sessions occurring 1-3 weeks apart: (1) smoking as usual and (2) overnight (12-14 h) abstinent. Both sessions occurred before 12 noon for standardization and the order of sessions was counterbalanced across participants. For both sessions, subjects were instructed to refrain from alcohol or other drugs for at least $24 \mathrm{~h}$ before the session, to abstain from food for $2 \mathrm{~h}$ before the session, and to abstain from caffeine for at least $1 \mathrm{~h}$ before the session. For the smoking as usual session, participants were asked to smoke one of their own brand cigarettes before entering the clinic.

After arrival to the clinic, participants completed self-report questionnaires (below) and provided a carbon monoxide (CO) breath sample. This sample was used to verify overnight abstinence for the abstinence session. All participants self reported no smoking for the past $14 \mathrm{~h}$. We used a criterion of $15 \mathrm{ppm}$, because reduced lung function in long term smokers produces variability in ability to clear CO overnight. All but two participants had a CO level below the criterion of $15 \mathrm{ppm}$; the other two participants had COs of 16 and 18 (both had reductions in CO of $>60 \%$ from the satiety to abstinent session; after reinterview, both indicated strongly that they had not smoked). CO level at the satiety session was also used to control for recent tobacco exposure in the analyses. All participants were fed a meal of a Nutri-Grain ${ }^{\circledR}$ bar and water to standardize food intake before scanning. After completion of these assessments and procedures, participants were walked to the radiology clinic $(\sim 20$ $\mathrm{min}$ ) for the scanning session. Including set-up time, there was a span of $\sim 45-60$ min between the last cigarette smoked (on the smoking as usual day) and the imaging session.

Measures. At baseline, participants completed standard demographic assessments and measures of smoking history. The Fagerstrom Test for Nicotine Dependence (Fagerstrom and Schneider, 1989), a six-item validated scale, was also administered. After arrival to the clinic, participants also rated the severity of their cravings to smoke using two four-point Likert scale items ("cravings for a cigarette" and "urges to smoke at this time"; scored from 0 , not present, to 3 , severe) that predicted smoking relapse in previous research (Killen and Fortmann, 1997). In addition, we assessed total withdrawal scores (Hughes et al., 1984; Cinciripini et al., 1995). On this 18-item scale, participants rate the severity of physical/ somatic (e.g., increased heart rate), cognitive (e.g., difficulty concentrat- ing), and affective (e.g., irritability) symptoms on a four-point Likert scale ( 0 , not present, to 3 , severe).

Imaging protocol. ASL perfusion MRI was used to characterize changes in resting regional blood flow. ASL perfusion MRI provides a noninvasive and comparatively inexpensive method for quantifying CBF during a task condition or a resting state using magnetically labeled arterial blood water as an endogenous tracer (Detre et al., 1992). The magnetic tracer has a decay rate of T1, the longitudinal relaxation time for blood water or brain tissue, which is sufficiently long ( $1-2 \mathrm{~s}$ at 3 tesla) to allow perfusion of the microvasculature to be detected. Tissue perfusion is quantified by pairwise comparison with separate images acquired with control labeling, using an appropriate model (Alsop et al., 2000). An unlimited number of CBF measurements can be made, enhancing power for time series based statistical analysis. CBF measurements with ASL perfusion MRI have been shown to agree with results from O-15 PET (Ye et al., 2000; Feng et al., 2004) and dynamic susceptibility contrast agent approaches (Siewert et al., 1997; Wolf et al., 2003). In addition, ASL perfusion measurements both at rest and during task activation have been demonstrated to be highly reproducible across intervals varying from a few minutes to 7 weeks (Floyd et al., 2001; Wang et al., 2003; Parkes et al., 2004; Hermes et al., 2007).

Imaging was performed on a Siemens (Erlangen, Germany) 3T Trio MR scanner using a product volume coil. High-resolution structural MR images were acquired in both sessions for CBF image spatial normalization, using a T1-weighted three-dimensional (3D) magnetizationprepared rapid acquisition gradient echo (MP-RAGE) sequence with 160 slices, $1.0 \mathrm{~mm}$ thickness, a $22 \mathrm{~cm}$ field of view (FOV), $192 \times 256$ matrix, an inversion time of $1100 \mathrm{~ms}$, repetition time (TR) of $1630 \mathrm{~ms}$, echo time (TE) of $3 \mathrm{~ms}$, and number of excitations equalling 1 . Immediately after the MP-RAGE scan, 40 resting label/control image pairs were acquired to measure CBF using an amplitude modulated continuous ASL (CASL) perfusion MRI sequence (Wang et al., 2005) with parameters as follows: labeling time, $2 \mathrm{~s}$; postlabeling delay, $1200 \mathrm{~ms}$; FOV, $22 \mathrm{~cm}$; matrix, $64 \times$ $64 \times 16$; flip angle, $90^{\circ}$; TR, $4 \mathrm{~s}$; TE, $17 \mathrm{~ms}$; slice thickness, $7 \mathrm{~mm}$; interslice spacing of $0.5 \mathrm{~mm}$. These data were acquired before a longer scanning protocol that included fMRI.

During the perfusion scan, participants were instructed to lie quietly in the scanner. Because we were interested in cravings during a resting state, and because cognitive tasks are known to change CBF (Xu et al., 2005), participants did not perform any task during the sessions. They were reminded not to fall asleep during the scan.

Data analysis. An SPM5 (Wellcome Department of Cognitive Neurology, London, UK) based ASL data processing toolbox, ASLtbx (Wang et al., 2007), was used for data analyses. For each session, ASL image pairs were realigned to the mean of all control images to correct for head movements, and spatially smoothed with a 3D isotropic Gaussian kernel with full-width at half-maximum of $10 \mathrm{~mm}$. One subject was excluded because of excessive motion, resulting in a sample size of 14 . CBF image series were generated using a simplified two-compartment CASL perfusion model (Wang et al., 2005) with the simple-subtraction method for CBF calculation (Aguirre et al., 2005). For each session, the mean control image was coregistered to the structural image acquired in the same session using the mutual information based coregistration algorithm provided by SPM 5 . The same coregistration parameters were also used to coregister the CBF maps to the structural image. The structural image was then spatially normalized to the MNI standard brain provided by SPM5. The same parameters were used to normalize the CBF maps to the MNI standard space. Each subject's normalized mean control images were segmented using SPM5. The segmented gray matter masks were averaged and the overlap of all subjects' gray matter was pulled out and used as a final mask for calculating global CBF of each session.

Contrast analysis between the abstinence and smoking session was conducted on each subject's normalized CBF images using a general linear model (first level analysis). A random effect analysis using onesample $t$ testing (Holmes and Friston, 1998) on the parametric maps of the first level contrast was used to generate a statistical parametric map of the $T$ statistic at each voxel for population inference (second-level analysis). Multiple regression analysis was conducted by including the firstlevel contrast analysis results and the changes of craving scores, $\mathrm{CO}$ scores of the smoking session, and gender to examine the corresponding 


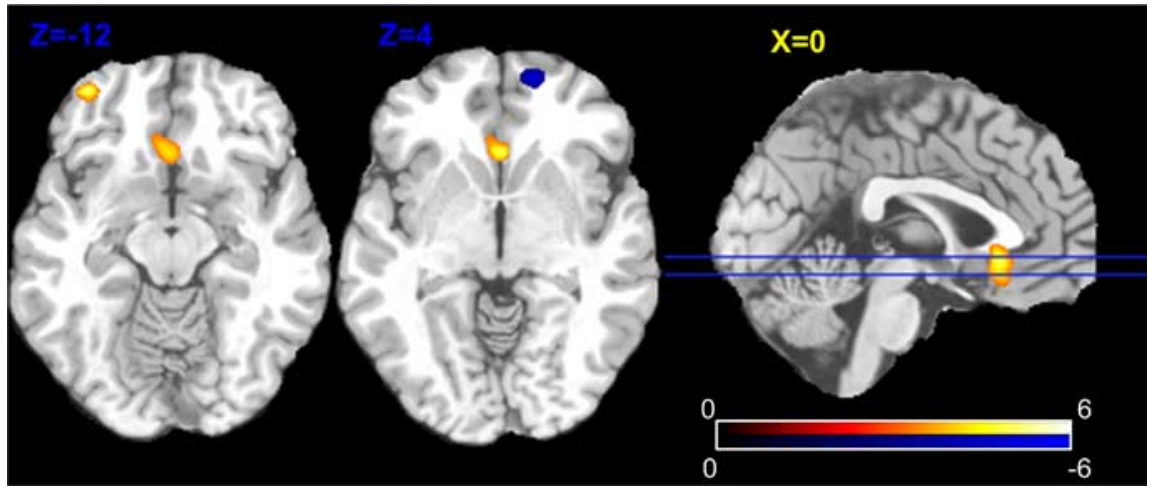

Figure 1. Statistical map of the regional CBF changes caused by abstinence (vs satiety). Yellow regions indicate greater CBF during abstinence; the blue region indicates a CBF decrease. The voxelwise statistical significance threshold is $p<0.005$ ( $t>3.17$, two tailed, uncorrected) and the color bar indicates the range of $t$ values displayed. The two blue lines superposed on the medial sagittal slice indicate the locations of the two left axial slices.

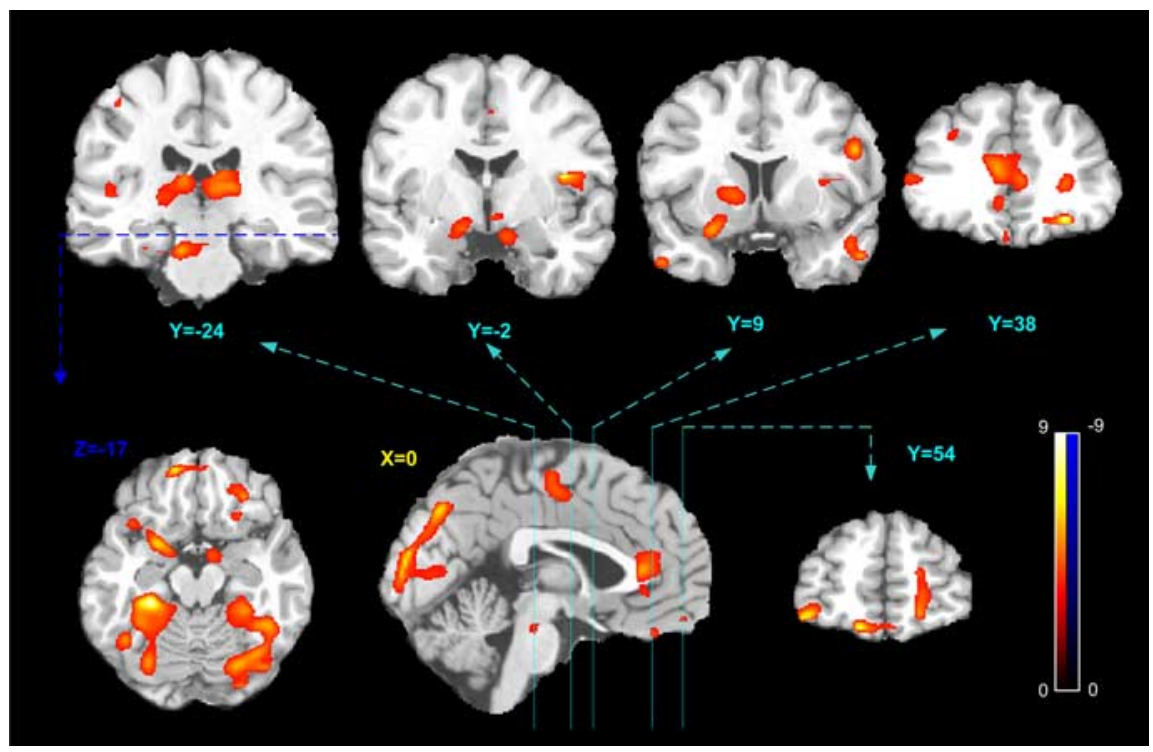

Figure 2. Association of abstinence-induced craving with regional CBF changes. The five coronal slices (4 in top row; 1 in bottom row) show suprathreshold craving-correlated CBF changes in bilateral thalamus, bilateral nucleus accumbens, right insula, bilateral medial temporal cortex, left caudate/putamen, bilateral dorsolateral prefrontal cortex, anterior cingulate cortex, and the orbitofrontal cortex. An axial slice (bottom row) shows suprathreshold clusters in the amygdala and the hippocampus. Suprathreshold correlation clusters in the occipital cortex, midbrain, and the anterior cingulate were also shown in the sagittal slice (bottom row). The voxelwise statistical significance threshold is $p<0.005$ ( $t>3.17$, two tailed, uncorrected) and the color bar indicates the range of $t$ values displayed.

correlations between the perfusion changes and the changes in cravings induced by abstinence (i.e., abstinence minus satiety). Another multiple regression with the total withdrawal score difference (abstinence minus satiety), CO scores of the smoking session, and gender as covariates was performed to examine correlations between CBF changes and changes in total withdrawal symptoms.

Two-sample $t$ tests were applied to test (1) whether there is a significant main effect of session (i.e., abstinence vs satiety state) on global CBF values, and (2) whether global CBF changes and changes in craving scores (abstinence vs satiety) are correlated. Gender and the CO scores of the smoking session were included as nuisance covariates.

Statistical analysis results were first thresholded with an uncorrected voxelwise threshold of $p<0.005$. Multiple comparison corrections were then performed using the familywise error (FWE) theory and based on the small volume correction (SVC) (Worsley et al., 1996). The small volume was chosen using a sphere located in the center of the corresponding regions.

\section{Results}

Descriptive data on study population

Of the 14 participants with useable imaging data, six $(43 \%)$ were male and eight $(57 \%)$ were female. Fifty percent of participants had at least some college education. The average age was $38.9(\mathrm{SD}, 10.4)$ and participants smoked, on average, 16.9 (SD, 5.6) cigarettes per day. The average Fagerstrom Test for Nicotine Dependence score was 4.93 (SD, 2.06) and all participants were of European ancestry.

As expected, the nicotine abstinence manipulation produced significant differences in cravings across the two sessions [average craving scores of 3.57 (SD, 2.06) vs 0.71 (SD, $0.91)$ for abstinence and smoking, respectively; $t=5.07 ; p=0.0001]$.

\section{Global CBF analysis of session effects and correlations with craving} A two-sample $t$ test comparing CBF during abstinence and satiety did not show a significant effect $(p=0.21$, with $\mathrm{CBF}=$ $56.6 \pm 2.9 \mathrm{ml} / 100 \mathrm{~g} / \mathrm{min}$ during abstinence and $53.4 \pm 2.2 \mathrm{ml} / 100 \mathrm{~g} / \mathrm{min}$ for satiety). However, the multiple regression analysis did show a significant $(p=0.022)$ positive correlation between the global CBF increases (abstinence vs satiety session) and abstinence-induced craving (abstinence vs satiety session).

Regional CBF analysis of session effects All imaging results are presented using the neurological orientation, with the left side of the brain on the left side of the image. $X, Y$, and $Z$ refer to the left-right (sagittal), anterior-posterior (coronal), and the inferior-superior (axial) orientations, respectively, and are color coded in yellow, turquoise, and blue. The location of each slice is marked by lines with different colors and the corresponding $X$ or $Z$ value.

Figure 1 shows the main regional CBF effects of abstinence versus satiety. At an arbitrary voxelwise threshold of $p<0.005$ (uncorrected for multiple voxels) and cluster size over 30, two hyperperfusion clusters were found in the ACC (the most inferior part) partly overlapping the medial OFC (hyperperfusion, $p=0.005$, FWE correction using SVC with $r=12 \mathrm{~mm}$ ), and in the left OFC ( $p=0.044$, FWE using SVC with $r=10 \mathrm{~mm}$ ); a hypoperfusion cluster (Fig. 1, the blue spot) was found in the right PFC ( $p=0.032$, FWE using SVC with $r=10 \mathrm{~mm})$. In these three suprathresholded clusters, $14 \mathrm{ml} / 100 \mathrm{~g} / \mathrm{min} \mathrm{CBF}$ increases were found in right $\mathrm{OFC}$ and ACC/medial OFC; a $6.7 \mathrm{ml} / 100 \mathrm{~g} / \mathrm{min} \mathrm{CBF}$ decrease was found in the left OFC.

Regional CBF analysis of abstinence-induced craving

Figure 2 shows the correlation between abstinence-induced absolute $\mathrm{CBF}$ changes and abstinence-induced craving changes (the difference in scores between abstinence and satiety sessions). Controlling for gender and CO values at the smoking session, 

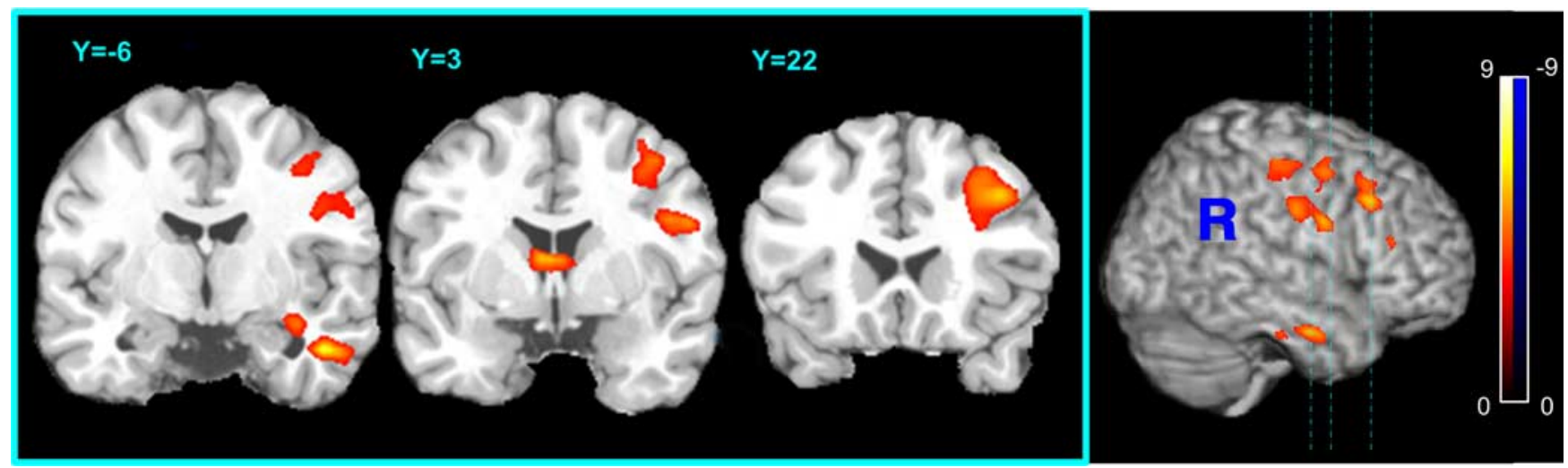

Figure 3. Association of abstinence-induced global withdrawal scores with regional CBF changes. Significant correlations of CBF change with change in withdrawal symptoms were found in the right dorsolateral prefrontal cortex, caudate, right hippocampus, and the right medial temporal gyrus. The voxelwise statistical significance threshold is $p<0.005$ ( $t>3.17$, two tailed, uncorrected) and the color bar indicates the range of $t$ values displayed.

significant correlation clusters were identified in the ACC $(p=$ 0.001 , FWE correction), left thalamus/hippocampus ( $p=1 \mathrm{e}-10$, FWE correction), right thalamus/hippocampus ( $p=1 \mathrm{e}-9$, FWE correction), right PFC ( $p=0.048$, FWE correction), and occipital cortex ( $p=1 \mathrm{e}-8$, FWE correction). Using SVC, additional significant correlation clusters were found in right amygdala/ ventral striatum $(p=0.016$, FWE correction with SVC; $r=10$ $\mathrm{mm})$, left amygdala/ventral striatum ( $p=0.005$, FWE correction with SVC; $r=10 \mathrm{~mm})$, left OFC ( $p=0.011$, FWE with SVC; $r=$ $10 \mathrm{~mm})$, medial OFC ( $p=0.032$, FWE with SVC; $r=10 \mathrm{~mm})$, right OFC ( $p=0.018$, FWE with SVC; $r=10 \mathrm{~mm}$ ), left dorsolateral prefrontal cortex (DLPFC) $(p=0.013$, FWE with SVC; $r=12 \mathrm{~mm})$, right DLPFC $(p=0.002$, FWE with SVC; $r=12$ $\mathrm{mm})$, left caudate/putamen ( $p=0.008$, FWE with SVC; $r=10$ $\mathrm{mm})$, right insula ( $p=0.013$, FWE with SVC; $r=10 \mathrm{~mm})$, left medial temporal gyrus (MTG) $(p=0.007$, FWE with SVC; $r=$ $12 \mathrm{~mm}$ ), and right MTG ( $p=0.01$, FWE with SVC; $r=12 \mathrm{~mm})$.

Based on a previous study (Tanabe et al., 2007), a region-ofinterest (ROI) analysis was also performed to examine the correlation between the CBF changes in thalamus and abstinence-induced cravings, controlling for CO level and gender. The thalamus ROI was provided by the Wake Forest Pick atlas utility (Maldjian et al., 2003) and covers the whole bilateral thalamus. This ROI-based multiple regression analysis yielded a significant ( $p=0.04$ ) positive correlation between the abstinence-induced perfusion increase and the subjective craving increases (abstinence minus satiety).

\section{Regional CBF analysis of abstinence-induced total withdrawal symptoms}

Figure 3 shows the correlation between abstinence-induced CBF changes and total withdrawal score changes (i.e., difference in scores between abstinence and satiety sessions). Three clusters showing significant correlations between $\mathrm{CBF}$ change and total withdrawal symptom change were identified: the right DLPFC ( $p=1 \mathrm{e}-6$, FWE correction), caudate $(p=0.024$, FWE using SVC; $r=12 \mathrm{~mm}$ ), and the right MTG/hypohippocampus ( $p=$ 0.0001 , FWE correction).

\section{Discussion}

The present study examined effects of abstinence from smoking on resting $\mathrm{CBF}$, as well as the correlation between abstinence-induced changes in resting $\mathrm{CBF}$ and changes in cravings to smoke. Abstinence (vs satiety) state effects on global CBF were not significant; however, abstinence state was associated with significant regional
CBF increases in ACC (the most inferior part) and medial OFC. Abstinence-induced cravings to smoke were correlated significantly with resting global CBF changes (abstinence vs satiety), as well as resting $\mathrm{CBF}$ changes in several ROIs: the right DLPFC, OFC, left inferior frontal cortex, occipital cortex, ACC, ventral striatum/nucleus accumbens, thalamus, amygdala, bilateral hippocampus, left caudate, right insula, and medial temporal gyrus. Consistent with our predictions, hyperperfusion associated with abstinence-induced craving was found in regions that are rich in $\alpha 4 \beta 2 \mathrm{nAChRs}$ (ventral striatum/nucleus accumbens, DLPFC, thalamus, inferior frontal cortex), regions linked with learning and memory processes (amygdala, hippocampus), and those involved in attention and behavioral control (ACC, OFC).

The ACC and OFC are paralimbic regions that have been implicated in drug-seeking behavior (Kalivas and Volkow, 2005), partly via their roles in reward-related decision-making and cognitive control (Rushworth et al., 2007). As with abstinence-induced craving in the present study, ACC and OFC activation have been linked with cue-induced craving in previous research (Brody et al., 2002; David et al., 2005; McClernon et al., 2005; Franklin et al., 2007). These same regions are activated after delivery of nicotine vs placebo (Stein et al., 1998; Rose et al., 2003), although decreased ACC activation has also been observed among smokers performing cognitive tasks (Ghatan et al., 1998; Ernst et al., 2001; Hahn et al., 2007). Together, these findings support an important role for the ACC and OFC in nicotine dependence, and perhaps specifically in craving responses that are known to promote relapse.

The hippocampus and amygdala, which play important roles in associative learning and synaptic plasticity (Tronson and Taylor, 2007), are also implicated in abstinence-induced cravings in the present study. These results are consistent with some smoking cueinduction studies (Due et al., 2002; Franklin et al., 2007). The ventral striatum, also associated with abstinence-induced craving in the present study, is activated by smoking related cues as well (David et al., 2005; Franklin et al., 2007). The ventral striatum has been implicated in the anticipation and immediate response to rewards, particularly among individuals scoring high on a trait measure of impulsivity (Hariri et al., 2006).

Correlations observed in the present study between abstinenceinduced cravings and insula activation are consistent with the role of this region in awareness of interoceptive cues (Damasio et al., 2000), which are a key component of the subjective nicotine withdrawal syndrome (Hughes et al., 1984). Furthermore, a previous study doc- 
uments that smokers with damage to the insula report greater ease of smoking cessation with minimal smoking urges, compared with smokers with damage to other brain regions (Naqvi et al., 2007).

Finally, the thalamus, rich in nAChRs that stimulate dopamine release (Brody et al., 2006), is also implicated by the present analysis of abstinence-induced craving. Previously, Tanabe and colleagues (Tanabe et al., 2007) reported an inverse correlation between abstinence-induced global withdrawal symptoms and reductions in $\mathrm{CBF}$ in the thalamus. In contrast, we observed a positive correlation between the increase of CBF in the thalamus during abstinence and the abstinence-induced craving. To address these differences, we also analyzed data on a global withdrawal symptom scale, finding CBF increases in the right DLPFC, hippocampus, and medial temporal gyrus, but not thalamus.

The greater sensitivity of craving scores, compared with global withdrawal scores, for correlations with CBF changes during abstinence is noteworthy. Increased cravings to smoke post cessation consistently predict relapse, particularly in the first weeks of quitting (Doherty et al., 1995; Swan et al., 1996; Killen and Fortmann, 1997; al'Absi et al., 2004). For example, in a sample of $>2600$ smokers, one third of those $(32 \%)$ who reported the highest levels of craving relapsed within 1 week of quitting, compared with $15 \%$ of those with the lowest levels of craving (Killen and Fortmann, 1997). Global assessments of withdrawal that encompass somatic, cognitive, and affective symptoms (Hughes, 2007) tend to be less robust predictors of relapse. For example, increased withdrawal symptoms have been shown to predict relapse during cessation treatment in some studies (Piasecki et al., 2000; al'Absi et al., 2004), but not others (Piasecki et al., 1997; Strasser et al., 2005). This is further supported by data showing that the extent to which smoking cessation treatments reduce withdrawal does not necessarily correlate with their efficacy (Jorenby et al., 1995). Thus, global measures of abstinence symptoms may not only be less sensitive measures in neuroimaging studies of smoking behavior, but also appear to have reduced prognostic value compared with measures of specific constructs (e.g., craving).

The present study has several strengths, including the withinsubject design and the abstinence manipulation. However, there are potential limitations as well. First, on the day of the satiety session, participants smoked their last cigarette 45-60 min before the scanning session. This time lag was introduced because smoking a cigarette immediately before the scan would make it difficult to disentangle the effects of acute nicotine delivery. Nonetheless, it is possible that some participants may have experienced minor abstinence symptoms during the smoking session. However, craving scores between the two sessions were significantly different, suggesting that the abstinence manipulation was effective. Second, we used CO to verify overnight abstinence because cotinine assays are not specific to recent tobacco use, although it should be noted that CO levels are most sensitive for recent smoking, rather than chronic smoking. Third, physiological assessments were not performed during scanning, and we cannot rule out the possibility that a subject fell asleep, despite our urging to the contrary. Fourth, with only two sessions examined in this study and no control group, it was not possible to determine the intersession test-retest reliability of ASL perfusion for our data. However, the effects reported in the present study were much larger than the mean differences observed between two time points 7 weeks apart in a previous CASL reproducibility study (Hermes et al., 2007). In future work, it would be desirable to use a design that would allow test-retest reliability to be determined.

It should also be noted that it is not possible from the present data to tease apart the effects of smoking abstinence versus nicotine abstinence on craving responses and associated regional brain activation. Non-nicotine aspects of smoking are an important component of dependence, and denicotinized cigarettes produce reduction in cravings (Rose, 2006). Furthermore, a single cigarette results in $88 \%$ occupancy of $\alpha 4 \beta 2$ nicotinic receptors $3 \mathrm{~h}$ after smoking (Brody et al., 2006), and nicotine can take several days to clear the brain (Staley et al., 2006). These data support the premise that abstinence-induced cravings arise from both nicotine and non-nicotine factors. Thus, it would be of interest for future studies to examine regional brain activation associated with craving reduction after nicotine versus denicotinized cigarettes.

The present findings contribute new evidence that abstinenceinduced (unprovoked) cravings to smoke are associated with increased activation in brain regions important in attention, behavioral control, memory, and reward. Items used to assess craving in the present study predict relapse in smoking cessation treatment (Killen and Fortmann, 1997). Thus, if validated in larger studies, these results may have important clinical implications. For example, perfusion MRI may aid in the identification of smokers at increased risk for relapse who may require more intensive therapy (Borsook et al., 2006).

\section{References}

Aguirre GK, Detre JA, Wang J (2005) Perfusion fMRI for functional neuroimaging. Int Rev Neurobiol 66:213-236.

al'Absi M, Hatsukami D, Davis GL, Wittmers LE (2004) Prospective examination of effects of smoking abstinence on cortisol and withdrawal symptoms as predictors of early smoking relapse. Drug Alcohol Depend 73:267-278.

Alsop DC, Detre JA, Grossman M (2000) Assessment of cerebral blood flow in Alzheimer's disease by spin-labeled magnetic resonance imaging. Ann Neurol 47:93-100.

Borsook D, Becerra L, Hargreaves R (2006) A role for fMRI in optimizing CNS drug development. Nat Rev Drug Discov 5:411-424.

Brody AL (2006) Functional brain imaging of tobacco use and dependence. J Psychiatr Res 40:404-418.

Brody AL, Mandelkern MA, London ED, Childress AR, Lee GS, Bota RG, Ho ML, Saxena S, Baxter Jr LR, Madsen D, Jarvik ME (2002) Brain metabolic changes during cigarette craving. Arch Gen Psychiatry 59:1162-1172.

Brody AL, Mandelkern MA, Lee G, Smith E, Sadeghi M, Saxena S, Jarvik ME, London ED (2004) Attenuation of cue-induced cigarette craving and anterior cingulate cortex activation in bupropion-treated smokers: a preliminary study. Psychiatry Res 130:269-281.

Brody AL, Mandelkern MA, London ED, Olmstead RE, Farahi J, Scheibal D, Jou J, Allen V, Tiongson E, Chefer SI, Koren AO, Mukhin AG (2006) Cigarette smoking saturates brain alpha 4 beta 2 nicotinic acetylcholine receptors. Arch Gen Psychiatry 63:907-915.

Caggiula AR, Donny EC, White AR, Chaudhri N, Booth S, Gharib MA, Hoffman A, Perkins KA, Sved AF (2001) Cue dependency of nicotine selfadministration and smoking. Pharmacol Biochem Behav 70:515-530.

Cinciripini PM, Lapitsky L, Seay S, Wallfisch A, Meyer III WJ, van Vunakis H (1995) A placebo-controlled evaluation of the effects of buspirone on smoking cessation: differences between high- and low-anxiety smokers. J Clin Psychopharmacol 15:182-191.

Conklin CA (2006) Environments as cues to smoke: implications for human extinction-based research and treatment. Exp Clin Psychopharmacol 14:12-19.

Damasio AR, Grabowski TJ, Bechara A, Damasio H, Ponto LL, Parvizi J, Hichwa RD (2000) Subcortical and cortical brain activity during the feeling of self-generated emotions. Nat Neurosci 3:1049-1056.

David SP, Munafo MR, Johansen-Berg H, Smith SM, Rogers RD, Matthews PM, Walton RT (2005) Ventral striatum/nucleus accumbens activation to smoking-related pictorial cues in smokers and nonsmokers: a functional magnetic resonance imaging study. Biol Psychiatry 58:488-494.

Detre JA, Leigh JS, Williams DS, Koretsky AP (1992) Perfusion imaging. Magn Reson Med 23:37-45.

Doherty K, Kinnunen T, Militello FS, Garvey AJ (1995) Urges to smoke during the first month of abstinence: relationship to relapse and predictors. Psychopharmacology (Berl) 119:171-178.

Due DL, Huettel SA, Hall WG, Rubin DC (2002) Activation in mesolimbic 
and visuospatial neural circuits elicited by smoking cues: evidence from functional magnetic resonance imaging. Am J Psychiatry 159:954-960.

Ernst M, Matochik JA, Heishman SJ, Van Horn JD, Jons PH, Henningfield JE, London ED (2001) Effect of nicotine on brain activation during performance of a working memory task. Proc Natl Acad Sci USA 98:4728 - 4733.

Fagerstrom KO, Schneider NG (1989) Measuring nicotine dependence: a review of the Fagerstrom Tolerance Questionnaire. J Behav Med 12:159-182.

Feng CM, Narayana S, Lancaster JL, Jerabek PA, Arnow TL, Zhu F, Tan LH, Fox PT, Gao JH (2004) CBF changes during brain activation: fMRI vs. PET. NeuroImage 22:443-446.

Floyd TF, Maldjian J, Gonzales-Atavales J, Alsop D, Detre JA (2001) Testretest stability with continuous arterial spin labeled (CASL) perfusion MRI in regional measurement of cerebral blood flow. Proc Intl Soc Magn Reson Med 9:1569.

Franklin TR, Wang Z, Wang J, Sciortino N, Harper D, Li Y, Ehrman R, Kampman K, O’Brien C P, Detre JA, Childress AR (2007) Limbic activation to cigarette smoking cues independent of nicotine withdrawal: a perfusion fMRI study. Neuropsychopharmacology 32:2301-2309.

Ghatan PH, Ingvar M, Eriksson L, Stone-Elander S, Serrander M, Ekberg K, Wahren J (1998) Cerebral effects of nicotine during cognition in smokers and non-smokers. Psychopharmacology (Berl) 136:179-189.

Hahn B, Ross TJ, Yang Y, Kim I, Huestis MA, Stein EA (2007) Nicotine enhances visuospatial attention by deactivating areas of the resting brain default network. J Neurosci 27:3477-3489.

Hariri AR, Brown SM, Williamson DE, Flory JD, de Wit H, Manuck SB (2006) Preference for immediate over delayed rewards is associated with magnitude of ventral striatal activity. J Neurosci 26:13213-13217.

Hermes M, Hagemann D, Britz P, Lieser S, Rock J, Naumann E, Walter C (2007) Reproducibility of continuous arterial spin labeling perfusion MRI after 7 weeks. Magma 20:103-115.

Holmes AP, Friston KJ (1998) Generalisability, random effects and population interference. NeuroImage 7:S754.

Hughes JR (2007) Effects of abstinence from tobacco: valid symptoms and time course. Nicotine Tob Res 9:315-327.

Hughes JR, Hatsukami DK, Pickens RW, Krahn D, Malin S, Luknic A (1984) Effect of nicotine on the tobacco withdrawal syndrome. Psychopharmacology (Berl) 83:82-87.

Jarvik ME, Madsen DC, Olmstead RE, Iwamoto-Schaap PN, Elins JL, Benowitz NL (2000) Nicotine blood levels and subjective craving for cigarettes. Pharmacol Biochem Behav 66:553-558.

Ji D, Lape R, Dani JA (2001) Timing and location of nicotinic activity enhances or depresses hippocampal synaptic plasticity. Neuron 31:131-141.

Jorenby DE, Smith SS, Fiore MC, Hurt RD, Offord KP, Croghan IT, Hays JT, Lewis SF, Baker TB (1995) Varying nicotine patch dose and type of smoking cessation counseling. JAMA 274:1347-1352.

Kalivas PW, Volkow ND (2005) The neural basis of addiction: a pathology of motivation and choice. Am J Psychiatry 162:1403-1413.

Killen JD, Fortmann SP (1997) Craving is associated with smoking relapse: findings from three prospective studies. Exp Clin Psychopharmacol 5:137-142.

Lerman C, Lesage MG, Perkins KA, O’Malley SS, Siegel SJ, Benowitz NL, Corrigall WA (2007) Translational research in medication development for nicotine dependence. Nat Rev Drug Discov 6:746-762.

Maldjian JA, Laurienti PJ, Kraft RA, Burdette JH (2003) An automated method for neuroanatomic and cytoarchitectonic atlas-based interrogation of fMRI data sets. NeuroImage 19:1233-1239.

McClernon FJ, Hiott FB, Huettel SA, Rose JE (2005) Abstinence-induced changes in self-report craving correlate with event-related FMRI responses to smoking cues. Neuropsychopharmacology 30:1940-1947.

Morissette SB, Palfai TP, Gulliver SB, Spiegel DA, Barlow DH (2005) Effects of transdermal nicotine during imaginal exposure to anxiety and smoking cues in college smokers. Psychol Addict Behav 19:192-198.

Naqvi NH, Rudrauf D, Damasio H, Bechara A (2007) Damage to the insula disrupts addiction to cigarette smoking. Science 315:531-534.

Nestler EJ (2002) From neurobiology to treatment: progress against addiction. Nat Neurosci 5 [Suppl]:1076-1079.

Niaura R, Abrams DB, Monti PM, Pedraza M (1989) Reactivity to high risk situations and smoking cessation outcome. J Subst Abuse 1:393-405.

Parkes LM, Rashid W, Chard DT, Tofts PS (2004) Normal cerebral perfusion measurements using arterial spin labeling: reproducibility, stability, and age and gender effects. Magn Reson Med 51:736-743.
Piasecki TM, Kenford SL, Smith SS, Fiore MC, Baker TB (1997) Listening to nicotine: negative affect and the smoking withdrawal conundrum. Phsychol Sci 8:184-189.

Piasecki TM, Niaura R, Shadel WG, Abrams D, Goldstein M, Fiore MC, Baker TB (2000) Smoking withdrawal dynamics in unaided quitters. J Abnorm Psychol 109:74-86.

Rose J (2006) Nicotine and nonnicotine factors in cigarette addiction. Psychopharmacoloy 184:274-285.

Rose JE, Behm FM, Westman EC, Mathew RJ, London ED, Hawk TC, Turkington TG, Coleman RE (2003) PET studies of the influences of nicotine on neural systems in cigarette smokers. Am J Psychiatry 160:323-333.

Rushworth MF, Buckley MJ, Behrens TE, Walton ME, Bannerman DM (2007) Functional organization of the medial frontal cortex. Curr Opin Neurobiol 17:220-227.

Shadel WG, Niaura R, Abrams DB, Goldstein MG, Rohsenow DJ, Sirota AD, Monti PM (1998) Scripted imagery manipulations and smoking cue reactivity in a clinical sample of self-quitters. Exp Clin Psychopharmacol 6:179-186.

Shiffman S, Engberg JB, Paty JA, Perz WG, Gnys M, Kassel JD, Hickcox M (1997) A day at a time: predicting smoking lapse from daily urge. J Abnorm Psychol 106:104-116.

Siewert B, Schlaug G, Edelman RR, Warach S (1997) Comparison of EPISTAR and $\mathrm{T} 2{ }^{*}$-weighted gadolinium-enhanced perfusion imaging in patients with acute cerebral ischemia. Neurology 48:673-679.

Staley JK, Krishnan-Sarin S, Cosgrove KP, Krantzler E, Frohlich E, Perry E, Dubin JA, Estok K, Brenner E, Baldwin RM, Tamagnan GD, Seibyl JP, Jatlow P, Picciotto MR, London ED, O’Malley S, van Dyck CH (2006) Human tobacco smokers in early abstinence have higher levels of $\beta 2^{*}$ nicotinic acetylcholine receptors than nonsmokers. J Neurosci 26:8707-8714.

Stein EA, Pankiewicz J, Harsch HH, Cho JK, Fuller SA, Hoffmann RG, Hawkins M, Rao SM, Bandettini PA, Bloom AS (1998) Nicotineinduced limbic cortical activation in the human brain: a functional MRI study. Am J Psychiatry 155:1009-1015.

Strasser AA, Kaufmann V, Jepson C, Perkins KA, Pickworth WB, Wileyto EP, Rukstalis M, Audrain-McGovern J, Lerman C (2005) Effects of different nicotine replacement therapies on postcessation psychological responses. Addict Behav 30:9-17.

Swan GE, Ward MM, Jack LM (1996) Abstinence effects as predictors of 28-day relapse in smokers. Addict Behav 21:481-490.

Tanabe J, Crowley T, Hutchison K, Miller D, Johnson G, Du YP, Zerbe G, Freedman R (2007) Ventral striatal blood flow is altered by acute nicotine but not withdrawal from nicotine. Neuropsychopharmacology, in press.

Tiffany ST, Cox LS, Elash CA (2000) Effects of transdermal nicotine patches on abstinence-induced and cue-elicited craving in cigarette smokers. J Consult Clin Psychol 68:233-240.

Tronson NC, Taylor JR (2007) Molecular mechanisms of memory reconsolidation. Nat Rev Neurosci 8:262-275.

Wang J, Aguirre GK, Kimberg DY, Roc AC, Li L, Detre JA (2003) Arterial spin labeling perfusion fMRI with very low task frequency. Magn Reson Med 49:796-802.

Wang J, Zhang Y, Wolf RL, Roc AC, Alsop DC, Detre JA (2005) Amplitudemodulated continuous arterial spin-labeling 3.0-T perfusion MR imaging with a single coil: feasibility study. Radiology 235:218-228.

Wang Z, Aguirre GK, Rao H, Wang J, Fernandez-Seara MA, Childress AR, Detre JA (2007) Empirical optimization of ASL data analysis using an ASL data processing toolbox: ASLtbx. Magn Reson Imaging, in press.

Wolf RL, Alsop DC, McGarvey ML, Maldjian JA, Wang J, Detre JA (2003) Susceptibility contrast and arterial spin labeled perfusion MRI in cerebrovascular disease. J Neuroimaging 13:17-27.

Worsley KJ, Neelin P, Vandal AC, Friston KJ, Evans AC (1996) A unified statistical approach for determining significant signals in images of cerebral activation. Human Brain Mapping 4:58-73.

Xu J, Mendrek A, Cohen MS, Monterosso J, Rodriguez P, Simon SL, Brody A, Jarvik M, Domier CP, Olmstead R, Ernst M, London ED (2005) Brain activity in cigarette smokers performing a working memory task: effect of smoking abstinence. Biol Psychiatry 58:143-150.

Ye FQ, Berman KF, Ellmore T, Esposito G, van Horn JD, Yang Y, Duyn J, Smith AM, Frank JA, Weinberger DR, McLaughlin AC (2000) $\mathrm{H}(2)$ (15)O PET validation of steady-state arterial spin tagging cerebral blood flow measurements in humans. Magn Reson Med 44:450-456. 